\title{
The influence of genetic selection on the milk fatty acid profile of spring calving dairy cows
}

\section{S Mc Parland ${ }^{1}$, D.P. Berry ${ }^{1}$, J Coleman ${ }^{1}$, R.F. Veerkamp ${ }^{4}$, H Soyeurt $^{2,3}$}

${ }^{1}$ Teagasc Moorepark Dairy Production Research Centre, Fermoy, Co. Cork, Ireland, ${ }^{2}$ Gembloux AgroBioTech - University of Liege, Animal Science Unit, Gembloux, Belgium, ${ }^{3}$ National Fund for Scientific Research, Brussels, Belgium,

${ }^{4}$ Wageningen University and Research Centre, Animal Breeding and Genomics, P.O. Box 65, 8200 AB Lelystad, Netherlands

Email: sinead.mcparland@teagasc.ie

Introduction Bovine milk represents a unique source of nutrients and bioactive components that act in synergy as well as independently and that can positively affect human health. However, the fatty acid (FA) content of bovine milk is not ideal, containing on average 65 to $70 \%$ saturated fat (SAT), 25 to 32\% monounsaturated fat (MONO), and 3 to $5 \%$ polyunsaturated fat (Soyeurt et al., 2006; Grummer, 1991). A more favourable combination for human health would be $30 \%$ saturated, $60 \%$ monounsaturated and $10 \%$ polyunsaturated fat. It is well known that the relative proportions of FA in milk can be altered through nutrition, but less is known on the potential of genetic selection for different FA composition. Of particular interest is the impact of current national breeding strategies on milk FA content. The objective of this study was to investigate the influence of genetic selection, using the Irish total merit index, the Economic Breeding Index (EBI) on milk FA throughout lactation.

Material and methods Three genetic groups of Holstein-Friesians (HF) were established from within the Teagasc Moorepark herd: a high genetic potential group of North American HF with an average EBI of $€ 77$ (HighNA; n=46), a group representing the Irish national average genetic potential of North American HF with an average EBI of $€ 49$ (LowNA; $\mathrm{n}=46$ ) and a group of high genetic potential New Zealand HF with an average EBI of $€ 89$ (HighNZ; n=48). Weekly milk samples collected from all cows for both AM and PM milk recordings between June 2008 and June 2009 were analysed using Mid-Infrared Spectrometry (MIR; Foss Milkoscan FT6000). The time period used in this study spanned over two experiments which investigated the impact of a) different concentrate feeding levels and grazing stocking rate, and b) different calving dates and grazing stock rates on overall performance on a basal grass based diet. Prediction equations previously derived and since validated on a selection of these animals (Soyeurt et al., 2010) were used to predict the FA composition of all milk samples using the MIR spectrum. The weighted average FA constituent was calculated for each week of lactation. A total of 4,135 records between days 5 and 305 of lactation from 140 cows remained. The effect of genetic selection on the content of SAT, unsaturated fat (UNSAT), MONO, short chain FA (aliphatic tails $<6$ carbons; SCFA), medium chain FA (aliphatic tails 6 - 12 carbons; MCFA) and long chain FA (aliphatic tails >12 carbons; LCFA) in milk fat was investigated using mixed linear models; cow was included as a random effect with a compound symmetry covariance structure assumed among records within cow. Experimental treatment, parity and week of lactation were included as fixed effects in all analyses.

Results Mean (standard deviation) SAT, UNSAT, MONO, SCFA, MCFA, LCFA (g/100g fat) across all three genetic groups was 66.2 (3.68), 35.7 (3.70), 29.8 (3.37), 9.4 (1.00), 46.6 (4.54), 44.4 (5.07), respectively. Mean (standard deviation) fat percentage for each of the groups was 4.5 (1.20) for the HighNA, 4.4 (1.16) for the LowNA and 4.8 (1.16) for the HighNZ. Experimental treatment had a significant effect on all FA investigated $(\mathrm{P}<0.01)$. The HighNZ cows produced more $(\mathrm{P}<0.05)$ SAT and less $(\mathrm{P}<0.05)$ UNSAT and MONO than either the HighNA or the LowNA groups $($ Table 1). The HighNZ cows also had higher $(\mathrm{P}<0.05)$ content of SCFA and MCFA in fat (Table 1). There was no difference between the high genetic merit and low genetic merit North American HF for any fat constituent. Although statistically significant, all differences reported are biologically small.

Table 1 Effect of genetic group on milk fat constituents ( $\mathrm{g} / 100 \mathrm{~kg}$ fat)

\begin{tabular}{lllllll}
\hline & SAT & UNSAT & MONO & SCFA & MCFA & LCFA \\
\hline HighNA & $66.1(0.31)^{\mathrm{a}}$ & $35.8(0.31)^{\mathrm{a}}$ & $29.9(0.27)^{\mathrm{a}}$ & $9.3(0.08)^{\mathrm{a}}$ & $46.5(0.32)^{\mathrm{a}}$ & $44.6(0.36)^{\mathrm{a}}$ \\
LowNA & $65.8(0.30)^{\mathrm{a}}$ & $36.1(0.31)^{\mathrm{a}}$ & $30.2(0.27)^{\mathrm{a}}$ & $9.3(0.08)^{\mathrm{a}}$ & $46.3(0.32)^{\mathrm{a}}$ & $45.0(0.36)^{\mathrm{a}}$ \\
HighNZ & $66.8(0.29)^{\mathrm{b}}$ & $35.1(0.29)^{\mathrm{b}}$ & $29.2(0.26)^{\mathrm{b}}$ & $9.6(0.07)^{\mathrm{b}}$ & $47.2(0.30)^{\mathrm{b}}$ & $43.7(0.34)^{\mathrm{b}}$ \\
P-value & $<0.01$ & $<0.01$ & $<0.01$ & $<0.001$ & $<0.05$ & $<0.01$ \\
\hline \hline
\end{tabular}

Conclusion These results indicate that genetic variation in milk FA composition exists and that HF cows of New Zealand ancestry have more SAT and less MONO and UNSAT per kg fat than HF cows of North American ancestry. In addition, within the North American animals, genetic selection for higher EBI does not appear to influence the FA profile of milk. Nonetheless, the routine availability of milk FA, predicted from MIR, facilitate the estimation of national breeding values for milk FA content thereby allowing the inclusion of milk quality in national breeding objectives.

Acknowledgements The RobustMilk project is financially supported by the European Commission under the Seventh Research Framework Programme, Grant Agreement KBBE-211708. The content of this paper is the sole responsibility of the authors, and it does not necessarily represent the views of the Commission or its services.

\section{References}

Grummer, R.R. 1991. Journal of Dairy Science 74, 3244-3257

Soyeurt, H., P. Dardenne, G. Lognay, D. Veselko, P. Mayeres, and N. Gengler. 2006. Journal of Dairy Science 89,36903695

Soyeurt, H., Mc Parland S., Berry D.P., Wall E., Gengler N., F. Dehareng, and P. Dardenne. 2010. Proceedings of the British Society of Animal Science, 13. 